\title{
The Possibility of Optical Analysis of the Mineral Fertilizers Physical Properties
}

\author{
D.V. YunOVIDOV ${ }^{a, b, *}$, V.V SOKOLOV ${ }^{a, b}$ AND A.S. BAKHVALOV ${ }^{c}$ \\ ${ }^{a}$ JSC "NIUIF", Cherepovets, Russia \\ ${ }^{b}$ Cherepovets State University, Cherepovets, Russia \\ ${ }^{c}$ JSC "Science Instruments", St. Petersburg, Russia
}

\begin{abstract}
The article describes the use of an optical surface recognition system for controlling physical parameters of mineral fertilizers produced on industrial scale. An optical device and an algorithm of extracting the properties from an RGB pixel matrix are described. On the basis of the received information, the possibility of classifying the physical parameters of the quality of fertilizers and their samples preparation (the size of the compressed particles, the grade and confirmation of preliminary drying execution) is shown. The possibility of the applying of this optical system as an independent device for quality control is proved.
\end{abstract}

DOI: 10.12693/APhysPolA.135.1108

PACS/topics: optical recognition, Random Forest classification, mineral fertilizers, data mining, image processing

\section{Introduction}

Modern industry is looking forward to simplifying and automating the quality control of products $[1,2]$. Rapid and multivariable methods of control are becoming more widespread. One of such methods is the X-ray fluorescence method of analysis (XRF). This method is a multi-element, sensitive, does not require complex sample preparation and can be applied in a wide range of concentrations. However, it strongly depends on the physical properties of the sample [3,4]: humidity, surface quality, aggregate state, etc. Without knowing these properties, $\mathrm{XRF}$ analysis is not accurate. To determine the physical properties of products, it is proposed to use the method of optical surface control. This system can be applied in addition to the XRF [5] and as an independent device.

\section{Experimental procedure}

In this article, an optical system installed in the dark room is introduced. It is equipped with a digital camera (resolution not less than $640 \times 480$, focal length 2.8 $12 \mathrm{~mm}$ and sensor type $1 / 2.7^{\prime \prime}$ CMOS) and LED lighting strip (wavelength $>370 \mathrm{~nm}$, light flux $\geq 50 \mathrm{~lm}$ ).

Mineral fertilizers of various types and grades are the object for the study. Thus, the type of fertilizers refers to the presence of certain macro and micro elements (NP, NPK, NPK $(\mathrm{S}), \mathrm{NP}(\mathrm{S}+\mathrm{S})+\mathrm{Zn}-$ contains sulfate and elemental sulfur and zinc). The fertilizer grade refers to the quantitative content of the components indicated in the type (12-52, 15-15-15, 4-30-15(16), 12-40(6+3)+1, respectively). Then, we use the term "grade" to mean the type and grade of fertilizer in general. Each sample was

*corresponding author; e-mail: dm.yunovidov@gmail.com prepared in the form of a "sandwich structure": pressed on a substrate of boric acid. Three types of particle size used: granules, powder fraction less than or equal to $500 \mu \mathrm{m}$ and the powder fraction less than or equal to $100 \mu \mathrm{m}$.

\section{Results and discussion}

Using the optical system, the surface of the entire set of complex phosphorus-containing fertilizers was studied. The image of each tablet was obtained at a fixed distance to the surface of the sample $( \pm 3 \mathrm{~mm})$. Furthermore, the area of the surface was selected with a resolution of at least $100 \times 100$ pixels in RGB format, converted to grayscale format, differentiated to eliminate the lighting trend and smoothed by a two-dimensional square median filter. This type of filter is chosen for its simplicity, efficiency and proper keeping of boundaries of brightness differences [6]. Thus, according to the obtained image, a "surface map" was constructed (Fig. 1).

Using the algorithm of "marching squares" [7], areas of bright and dark pixels - "anomalies" were allocated. The average brightness of pixels, the average area (relative to image area) and the number of "anomalies" were considered as features.

The configurable parameters of the described approach are: the $2 \mathrm{D}$ window for the median filter (from 1 to 30 pix) and the "contour constant" (from 0.1 to 2.0) the parameter of the algorithm of "marching squares", which determines the relative area of a single fragment in the image. As a criterion of quality, the Pearson linear correlation was applied between the calculated relative area of the "anomalies" and the particle size type (the size values were replaced by the numerical equivalents: 100,500 , and 5000). The best correlation is achieved with the window size of 10 pixels for median filter and the constant of 0.7 for "marching squares" algorithm. It was noticed that with a constant greater than 1.0 the correlation is improved. However, in this case, the values 

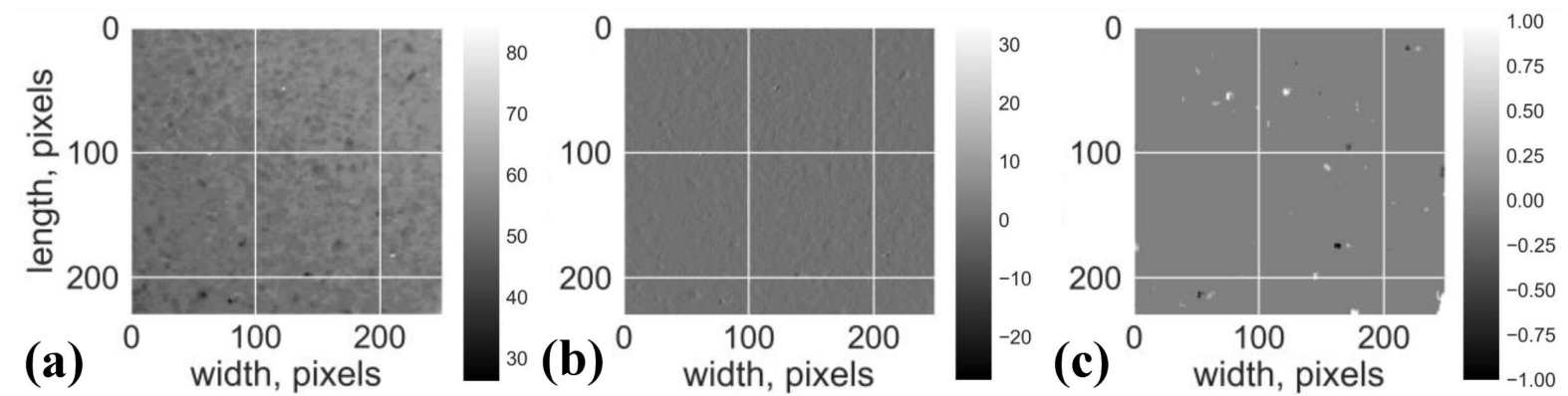

Fig. 1. Construction of a "surface map" for pressed powder $\leq 500 \mu \mathrm{m}$ of $\mathrm{NP}(\mathrm{S}+\mathrm{S})+\mathrm{Zn} 12-40(6+3)+1$ fertilizer. Surfaces: (a) — original, (b) - after differentiation, (c) — after smoothing by a median filter. Color-map indicates the intensity of pixels in grayscale (brightness).

Results of "Random Forest" classification.

TABLE I

\begin{tabular}{c|c|c|c|c|c|c}
\hline \hline \multirow{2}{*}{ Properties } & \multicolumn{2}{|c|}{ Precision } & \multicolumn{2}{c|}{ Recall } & \multicolumn{2}{c}{ F-metric } \\
\cline { 2 - 6 } & Average, \% & STD, \% & Average, \% & STD, \% & Average, \% & STD, \% \\
\hline dry condition* & 63.96 & 6.19 & 64.42 & 6.58 & 63.50 & 6.21 \\
particle size & 93.65 & 3.16 & 92.70 & 3.19 & 92.70 & 3.18 \\
grade & 61.79 & 3.93 & 59.07 & 3.09 & 59.01 & 2.52 \\
\hline
\end{tabular}

*binary properties: 0 - not dry, 1 - dry condition.

of the selected properties for the fraction less than or equal to $100 \mu \mathrm{m}$ are zeroed for many fertilizer grades and this fraction is not considered in calculation. The improvement in the correlation is observed due to the reduction of number of objects. But for the test samples the results will be worse. Thus, it was determined that the "contour constant" should not exceed the value in 1.0.

Using the "Random Forest" classification algorithm (standard parameters, the Python 2.7 programming language [8]), the possibility of predicting the physical properties of fertilizers according to the selected characteristics was evaluated (Table I). The evaluation was carried out by stratified cross-validation strategy using ten folds of not involved samples.

The obtained results show that the optical method is informative and can be used both as a stand-alone device (for particle size) and for supplementing other methods for monitoring quality parameters.

\section{Conclusion}

To sum up, the algorithm for extracting features from an RGB pixel matrix is described. Possibility of classifying the physical parameters of the quality of fertilizers and their samples preparation (the size of the compressed particles, the grade of fertilizer and confirmation if preliminary drying was done or not) is shown. Suitability of the described system as an independent device for calculating the particle size of objects (precision is 94\%) and assuming of the grade and humidity of the samples (precision more than $62 \%$ ) was carried out. However, in order to determine properly the last two parameters, an additional source of information is required, for example, the energy dispersive X-ray fluorescence analysis.

\section{Acknowledgments}

Special thanks to Ivanka Yunovidova for her help in writing the article.

\section{References}

[1] M. Offroy, L. Duponchel, Anal. Chim. Acta. 910, 1 (2016).

[2] A. Remes, K. Saloheimo, S.L. Jämsä-Jounela, Miner. Eng. 20, 1055 (2007).

[3] J. Hasikova, A. Sokolov, V. Titov, A. Dirba, Procedia Eng. 83, 455 (2014).

[4] D.V. Yunovidov, V.V. Sokolov, A.S. Bakhvalov, Diagn. Mater. 83, 15 (2017) (In Russian)

[5] D. Yunovidov, V. Sokolov, A. Bahvalov, in: 11 Winter School of Chemometrics (WSC 11), Saint Petersburg 2018 , p. 50.

[6] V. Strugailo, Sci. Educ. Bauman MSTU 12, 270 (2012).

[7] skimage.measure.find_contours, module documentation, Python code, scikit-image development team.

[8] randomforestclassifier, module documentation, Python code, scikit-learn developers team. 